\title{
Odontoma serie de casos. Revisión de literatura
}

\section{Odontoma case series. Review of literature}

\author{
Harris Ricardo J*, Rebolledo Cobos $M^{* *}$, Díaz Caballero A***, \\ Carbonell Muñoz Z***
}

\section{RESUMEN}

El odontoma es el tumor odontogénico más frecuente en cavidad oral, compuesto por una mezcla de células odontogénicas formando dentina y esmalte, presenta células y tejidos normales pero de estructura defectuosa, estos tejidos se encuentran en el área del cuerpo donde se presenta su crecimiento, al examen radiográfico se puede presentar imágenes radiopacas como un gran número de dientes rudimentarios llamados dentículos, en este caso se conoce como odontoma compuesto o presentarse imagen radiopaca como conglomeraciones amorfas, recibiendo el nombre de odontoma complejo. Su etiología se le relaciona con restos paradentales de Malassez, procesos inflamatorios, traumatismos, se le asocia en algunos casos con el quiste odontogénico calcificante, son asintomáticos, producen retención dentaria. El tratamiento es quirúrgico y su recurrencia es baja. Se reporta serie de casos que acudieron al servicio de estomatología y cirugía oral de la Universidad de Cartagena con impresión diagnostica de odontoma, se realizaron los procedimientos quirúrgicos confirmando los diagnósticos con los reportes histopatológicos.

Palabras clave: Odontoma, odontoma compuesto.

\section{SUMMARY}

Odontoma is the most frequent odontogenic tumor in oral cavity, comprising a mixture of odontogenic cells forming dentin and enamel. It shows cells and normal tissues but in abnormal structure. These tissues are located in the area of the body where it presents its growth. Under radiographic examination may be radiopaque images as a large number of rudimentary teeth called denticles, in this case is known as compound odontoma or presented as conglomerates amorphous radiopaque image, receiving the name of complex odontoma. Its etiology is related to remnants of Malassez paradontals, inflammatory processes. Trauma is sometimes associated with calcifying odontogenic cyst. Generally are asymptomatic, produce tooth retention. Treatment is surgical and recurrence is low. Is reported in the present paper a number of cases that went to the service of dentistry and oral surgery at the University of Cartagena with presumptive diagnosis of odontoma. Surgical procedures were performed to confirm the diagnosis with histopathological analysis.

Key words: Odontoma, compound odontoma.

Fecha de recepción: 5 de junio de 2009.

Aceptado para publicación: 20 de junio de 2009.

* Odontólogo. Universidad del Sinú Seccional Cartagena. Residente Estomatología y Cirugía Oral. Universidad de Cartagena.

** Odontóloga. Fundación Universitaria San Martín Sede Caribe. Residente de Estomatología y Cirugía Oral. Universidad de Cartagena.

*** Odontólogo. Universidad de Cartagena. Profesor titular. Facultad de Odontología. Universidad de Cartagena.

Harris Ricardo J, Rebolledo Cobos M, Díaz Caballero A, Carbonell Muñoz Z. Odontoma serie de casos. Revisión de literatura. Av. Odontoestomatol 2011; 27 (1): 25-32. 


\section{INTRODUCCIÓN}

Los odontomas son tumores odontogénicos mixtos, compuestos por una mezcla de células odontogénicas epiteliales y mesenquimatosas diferenciadas, comprenden tejido pulpar, esmalte, dentina, cemento, de aspecto normal pero con una estructura generalmente defectuosa. Estos tejidos y células se encuentran en el área del cuerpo donde se presenta su crecimiento, razón por la cual se les considera como hamartomas y no como neoplasias (1-3). Diversos autores escribieron sobre la clasificación de los odontomas, Sutton los divide en odontomas quísticos y sólidos, Bhaskar los clasifica en compuestos, complejos y quísticos. En 1978 la organización mundial de la salud (OMS) los clasificó en compuestos y complejos teniendo en cuenta la organización y el grado de alteración en la morfodiferenciación de las células odontogénicas $(4,5)$. El odontoma compuesto está formado por gran número de dientes pequeños de fácil identificación, conocidos como dentículos, se localizan con mayor frecuencia en la zona anterior del maxilar (6-8), entre las raíces y sobre la corona de un diente retenido. En el examen radiográfico se observa imagen radiolúcida con bordes definidos, múltiples zonas radio opacas en su interior, bien diferenciados, pueden provocar expansión de las cortical ósea, es asintomático, de crecimiento lento (9-11).

El odontoma complejo presenta conglomeraciones amorfas de tejido odontogénico que a diferencia del compuesto, no es fácil su diferenciación, al examen radiográfico, la imagen de este odontoma se observa como una masa amorfa radiopaca, única o múltiple, irregular, difusa, con un halo radiolúcido, se le localiza con mayor frecuencia en la zona posterior mandibular (12-14). Amado et al, en 2003; Junquera et al, en 2005; Ilief et al, en 2008 y Serra et al, en el 2009, reportan sobre odontomas transmucosos o periféricos de los tejidos blandos, que alcanzan a perforar la encía y erupcionar (15-18).

Existen otras patologías asociadas con odontomas como los quistes. En un estudio realizado por Hirshberg el cual reveló que el $24 \%$ de los pacientes, el odontoma era el tumor odontogénico más común, asociado a quiste odontogénico calcificante, Gallana et al, en 2005 reporta un casos, en el que se rebeló la asociación del quiste odontogénico calcificante con odontoma complejo y Delgado et al, en 2006 reporta sobre tumor odontogénico con presencia de tejido dental calcificado correspondiente a odontoma compuesto y complejo (19-21). La etiología del odontoma se le relaciona con restos paradentales de Malassez, procesos inflamatorios, traumatismos, hiperactividad odontoblástica, anomalías hereditarias como el síndrome de Gardner y Hermann (22-24).

Se deben incluir diagnósticos diferenciales ante imágenes radiográficas como un cementoma, osteomielitis esclerosante focal, diente supernumerario, tumor odontogénico epitelial calcificante, tumor odontogénico adenomatoide (25-27).

En la histopatología las células de los tejidos del odontoma son normales, se caracteriza por presentar dentina primaria, esmalte calcificado e hipocalcificado, cemento inmaduro y una cápsula, se presenta un desorden en las moléculas extracelulares de la matriz en le mesénquima (28-30). Estas lesiones se descubren con el examen radiográfico de rutina (3133), el motivo de consulta más habitual es la falta de erupción de un diente permanente, con o sin la persistencia del diente temporal (34-36). El Tratamiento para el odontoma es quirúrgico incluyendo enucleación, eliminando la cápsula de tejido conjuntivo que lo rodea (37-40).

La recurrencia de los odontomas es muy rara, en niños en que sus edades oscilan entre 1 y 5 años, no es recomendable realizar procedimientos quirúrgicos para su enucleación, ya que los odontomas se encuentran en estadios iníciales o tempranos del desarrollo, encontrándose porciones celulares no calcificadas de éste, que aumentan el riesgo de recurrencia cuando se realice la cirugía $(20,21)$.

\section{PRESENTACION DE CASOS}

\section{Caso 1}

Paciente femenina de 30 años de edad, remitida al servicio de estomatología y cirugía oral, por presentar expansión de la cortical ósea mandibular izquierda. La paciente refiere un aumento en el crecimiento 


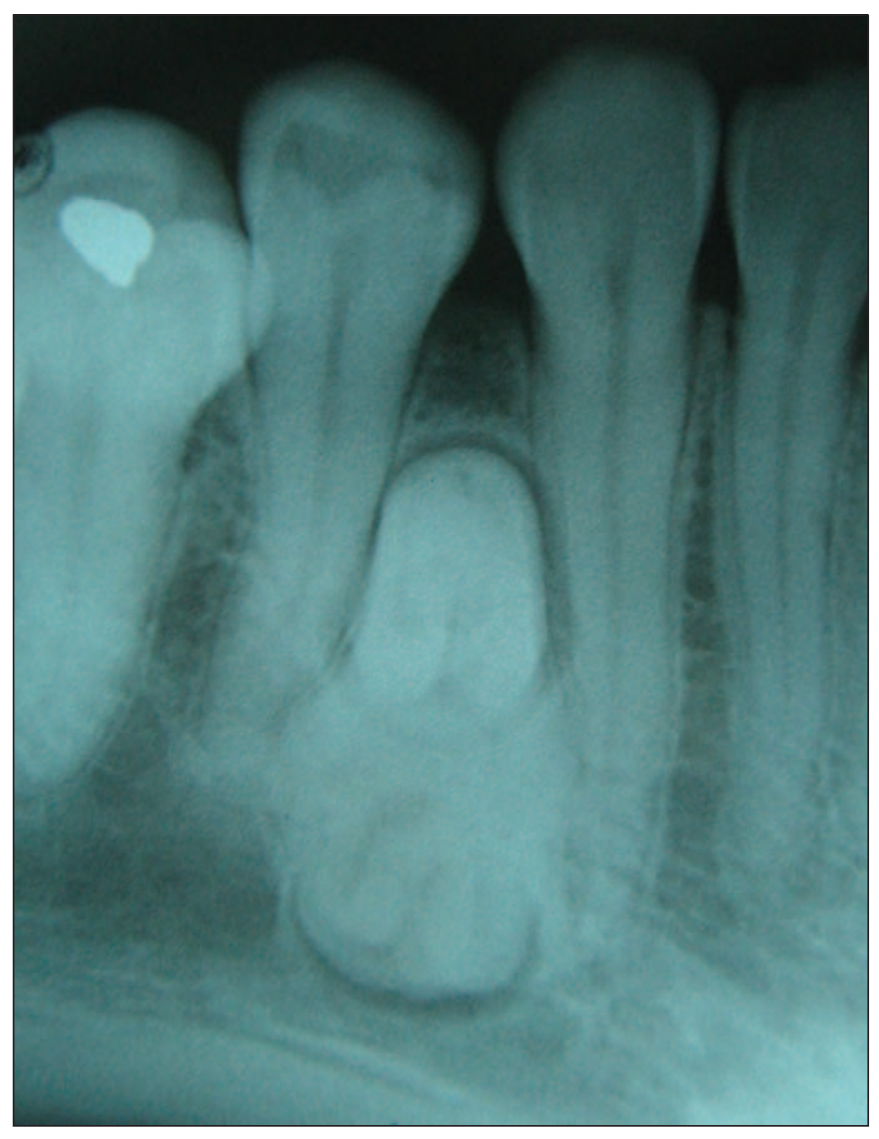

Fig. 1. En radiografía periapical se observa imagen radiopaca con bordes radiolúcidos bien definidos, localizada entre los órganos dentarios 33 y 34, con múltiples imágenes radiopacas en su interior de tamaño variable oscilando entre 2 y $4 \mathrm{~mm}$ de diámetro.

paulatino en la zona del canino izquierdo de la mandíbula, desde hace aproximadamente 6 años, asintomático, no interfiere con sus labores cotidianas,

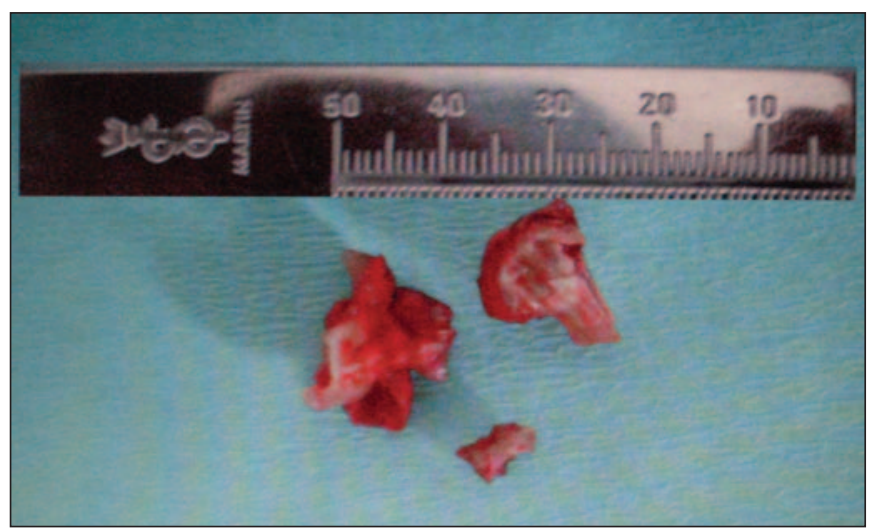

Fig. 2. Muestra del tejido extraído. no consumió medicamentos para reducir el tamaño de la lesión. En los antecedentes familiares la madre presenta diabetes, los antecedentes personales no son relevantes para el caso, en la exploración de órganos y sistemas no presenta alteraciones considerables.

Al examen intraoral presentó lesión tumoral, localizada en tabla ósea vestibular externa mandibular izquierda, de consistencia pétrea, base sésil, $5 \mathrm{~mm}$ diámetro y de $4 \mathrm{~mm}$ de altura, a nivel de los órganos dentarios 33 y 34, bordes definidos, del mismo color de la mucosa adyacente, asintomática.

En examen radiográfico se observó imagen radiopaca de $9 \mathrm{~mm}$ de diámetro con bordes radiolúcidos bien definidos, localizada entre los órganos dentarios 33 y 34, acompañada de múltiples imágenes radiopacas en su interior de tamaño variable oscilando entre los 1 y 3 mm. Impresión clínica de odontoma compuesto.

\section{Procedimiento}

Previo consentimiento informado, se aplicó técnica anestésica mandibular (lidocaína con epinefrina 2\%), el acto seguido consistió en la incisión festoneada, comprometiendo los órganos dentarios 32, 33, 34 y 35 , con descarga anterior distal del órgano dentario 32 (colgajo triangular de Reinmoller), luego se realizó levantamiento de colgajo mucoperióstico, se tuvo en cuenta las estructuras nerviosas que emergen por el agujero mentonero para protegerlas del trauma en el acto quirúrgico, posteriormente se realizó osteotomía para exponer la lesión tumoral, se continuó con la enucleación de la misma, luego se reposicionó colgajo para finalmente confrontar los bordes con puntos simples e interpapilares por medio de sutura no reabsorbible (seda 3-0). Se ordena farmacoterapia con Clindamicina y Nimesulide e indicaciones postoperatorias. Se realiza control postquirúrgico a los siete días, observándose tejidos blandos en buen estado y en proceso de cicatrización, el reporte histopatológico confirma diagnostico de odontoma, en el control radiográfico realizado a los 30 días después del procedimiento quirúrgico se observó imagen radiolúcida con zonas radiopacas evidenciando buen proceso de cicatrización óseo. 


\section{Caso 2}

Paciente masculino de 59 años que acude por presentar lesión tumoral en el maxilar superior del lado izquierdo, el paciente comenta presentar sintomatología dolorosa a la masticación, dos meses de evolución sin recibir tratamiento previo. En los antecedentes familiares padre presenta hipertensión, los antecedentes personales no son relevantes para el caso, en la exploración de órganos y sistemas no presenta alteraciones considerables.

En el examen clínico intraoral se evidenció lesión tumoral ulcerovegetante de $2 \mathrm{~cm}$ por $2 \mathrm{~cm}$ de diámetro, ubicado en maxilar superior del lado izquierdo a nivel de órganos dentales 23, 24 y 25, los cuales se encuentran ausentes clínicamente, de consistencia firme a la palpación con áreas blandas, color rojo, sintomático.

Al examen radiográfico se observó imagen radiopaca con bordes radiolúcidos bien definidos, localizada entre los órganos dentarios 23, 24 y 25 que se encuentran incluidos, con múltiples imágenes radio opacas en su interior de tamaño variable oscilando entre los 1 y $5 \mathrm{~mm}$ de diámetro. Impresión clínica de odontoma compuesto.

Se realizó el procedimiento quirúrgico de acuerdo al protocolo presentado en el caso anterior. El reporte histopatológico reportó odontoma compuesto; se realizó control 7 días después del procedimiento

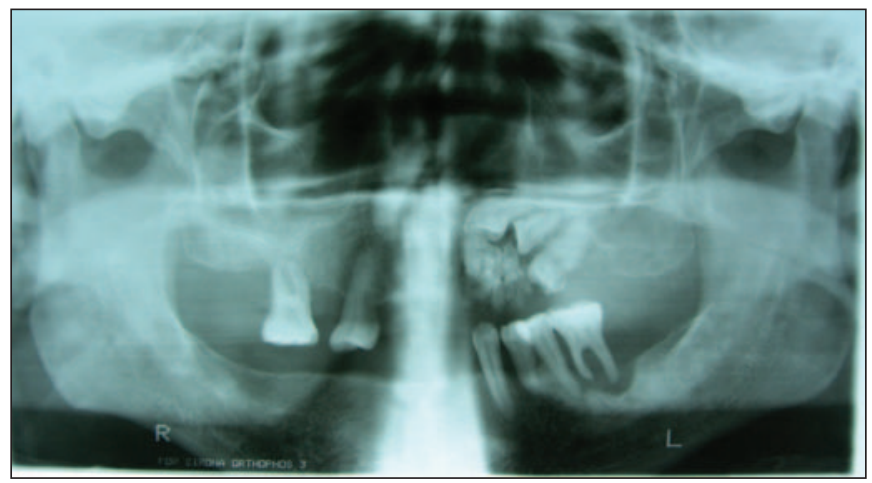

Fig. 3. En radiografía panorámica se observa imagen radiopaca con bordes radiolúcidos bien definidos, localizada entre los órganos dentarios 23, 24 y 25, con múltiples imágenes radiopacas en su interior de tamaño variable oscilando entre los 2 y 5 mm de diámetro.

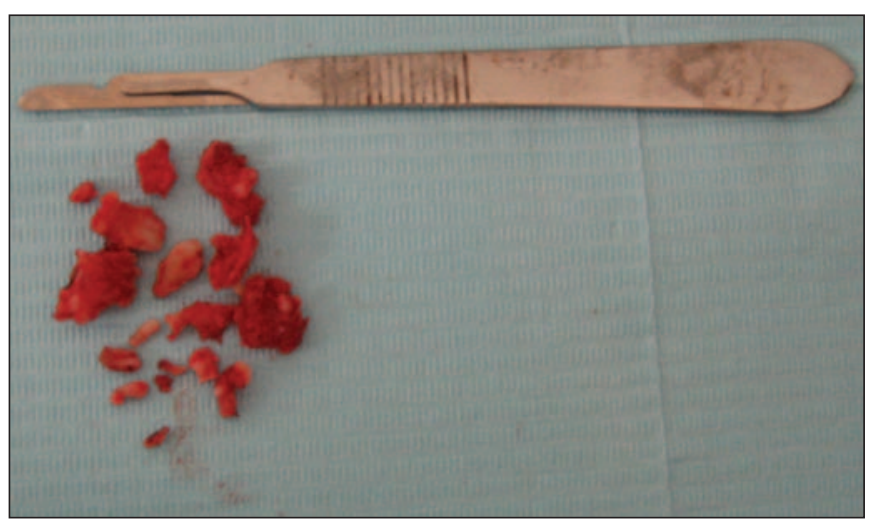

Fig. 4. Muestra del tejido extraído, se observan múltiples dentículos.

quirúrgico, observándose tejidos blandos en buen estado y en proceso de cicatrización.

\section{Caso 3}

Paciente femenina de 8 años de edad, remitida al servicio por presentar ausencia clínica de órgano dentario anteroinferior. Los antecedentes personales y familiares no son relevantes para el caso, en la

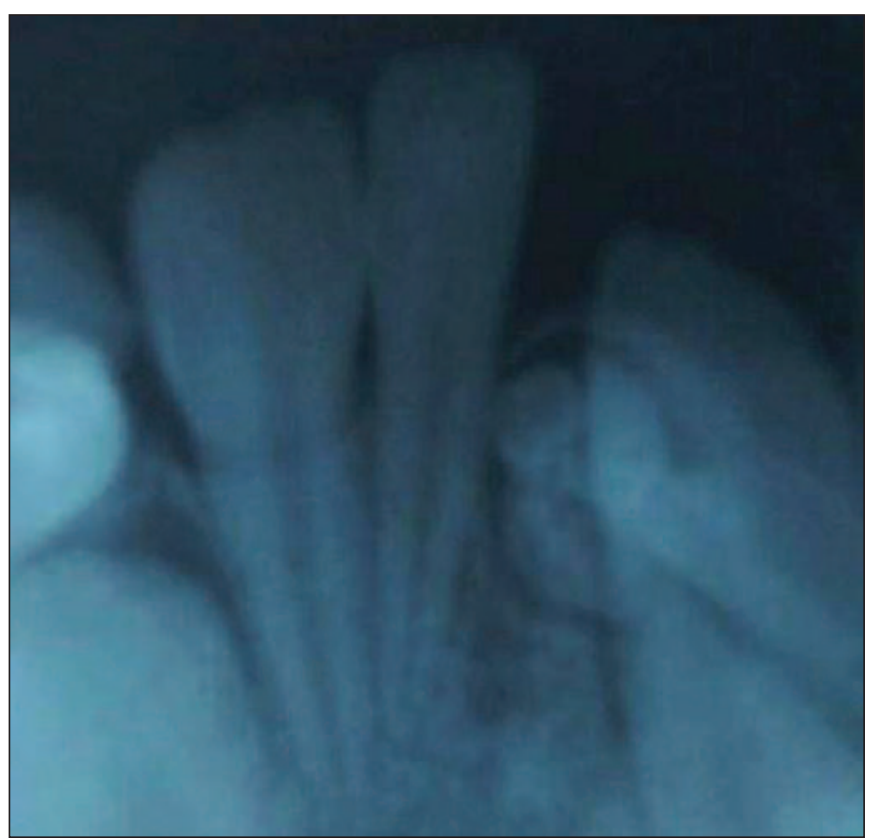

Fig. 5. En radiografía periapical se observa imagen radiopaca con bordes radiolúcidos bien definidos, localizada entre los órganos dentarios 31 y 41, con múltiples imágenes radiopacas en su interior de tamaño variable oscilando entre 2 y $4 \mathrm{~mm}$ de diámetro. 


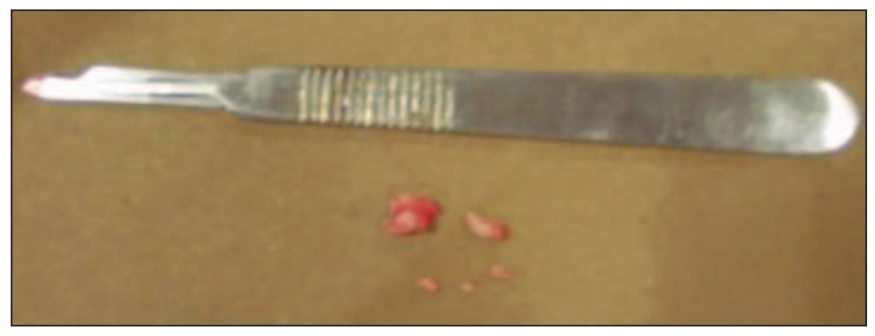

Fig. 6. Muestra del tejido extraído, se observan múltiples dentículos.

exploración de órganos y sistemas no presenta alteraciones considerables.

En el examen intraoral se observó lesión tumoral, de $2 \mathrm{~cm}$ de diámetro, localizada en mandíbula, expandiendo tabla vestibular externa, en zona de órganos dentarios 41 y 42, del mismo color de la mucosa adyacente, base sésil, superficie lisa, consistencia pétrea a la palpación, asintomática y de 7 meses de evolución.

Radiográficamente se observó imagen radiopaca con bordes radiolúcidos bien definidos, localizada entre los órganos dentarios 31 y 41, con múltiples imágenes radiopacas en su interior de tamaño variable oscilando entre los 2 y $4 \mathrm{~mm}$. Impresión clínica de odontoma compuesto.

Se realizó el procedimiento quirúrgico de acuerdo al protocolo presentado en el caso A. El reporte histopatológico reportó odontoma compuesto; se realizó control 7 días después del procedimiento quirúrgico, observándose tejidos blandos en buen estado y en proceso de cicatrización.

\section{DISCUSIÓN}

El odontoma es el tumor más frecuente de los tumores odontogénicos. Buchner et al, en el 2006 examinó una muestra de 1.088 tumores odontogénicos, en los cuales los odontomas representaron el $75,9 \%$ del total de la muestra (35); Ochsenius et al, en 2002 analizaron una muestra de 362 tumores odontogénicos, en los cuales los odontomas representaron 44,7\% (33), da Silva et al en un estudio publicado en el 2009 reportó que el odontoma es el tumor odontogénico más frecuente con un porcentaje de $73,9 \%$ (29)
En relación con la frecuencia de los diversos tipos de odontomas, Hidalgo et al, en un meta análisis, con una muestra 1.340 casos clasificados, encontró que el $61,3 \%$ eran odontomas compuestos y odontomas complejos el 37\%, presentándose esta relación en dos casos de tres presentados, la región anatómica más implicada, es el sector anterosuperior, seguido en el orden decreciente por la zona anteroinferior y la zona posteroinferior, en 1.000 casos relacionados el $40,5 \%$ se presento en mandíbula coincidiendo con dos casos presentados y en 1045 casos registrados el $42,9 \%$ no presento sintomatología coincidiendo con los tres casos de esta serie (12).

Hisatomi et al, en el 2002 analizaron 107 casos de odontoma en el cual, la mayor incidencia es en mujeres, presentándose esta relación en la serie de casos en dos pacientes femeninas (8), diversos autores refieren que la edad es un factor no determinante y se pueden identificar a cualquier edad, Concordando con esta serie de caso $(4,13,35)$.

Al examen histológico un estudio realizado por Chang et al, en 2003 con una casuística de 81, reveló la presencia de esmalte dental en $100 \%$, de cemento en el $88 \%$, de tejido pulpar en el $96 \%$, la cápsula fibrosa en el $93 \%$, las células del fantasma en el $83 \%$, coincidiendo con los componentes celulares reportados en la descripción microscópica de los tres casos presentados (24).

La razón de la consulta más frecuente es el retraso en la erupción dental permanente, asociada o no a la persistencia del diente temporal, o a la presencia de los diastemas (30). El tratamiento es la enucleación de la lesión, es muy importante retirar la cápsula que le rodea ya que si se dejan porciones de ésta aumenta el riesgo de recurrencia, en esta serie de casos no se presento recurrencia; el odontoma puede interferir con la erupción del diente permanente, desplazar los dientes adyacentes o dar lugar a un quiste dentígero $(13,21)$. El pronóstico de estos tumores es generalmente muy favorable $(15,35)$.

\section{BIBLIOGRAFÍA}

1. Oremchhingp Z, Joseph T, Varma B, Mungara J. A compound composite odontoma associated 
with unerupted permanent incisor, A Case Report. J Indian Sot Ped Prev Dent 2004 Sep;22(3): 114-7.

2. Ragalli CC, Ferreira JL, Blasco F. Large erupting complex odontoma. Int J Oral Maxillofac Surg 2000;29:373-4.

3. Philipsen HP, Reichart PA, Praetorius F. Mixed odontogenic tumours and odontomas. Considerations on interrelationship. Review of the literature and presentation of 134 new cases of odontomas. Oral Oncol 1997Mar;33(2):86-99.

4. de Olivera BH, Campos V, Marçal S. Compound odontoma-diagnosis and treatment: three case reports. Pediatr Dent 2001 Mar-Apr;23(2):151-7.

5. Da Costa C, Torriani D, Torriani M, da Silva R. Central incisor impacted by an odontoma. J Contemp Dent Pract 2008 Sep;9(6):122-8.

6. Ferrer MJ, Silvestre FJ, Estelles E, Grau D. Infección recurrente de un odontoma tras su apertura a la boca. Med Oral 2001;6:269-75.

7. Shelton JT, Owens BM, Schuman NJ. Compound Odontoma Associated Wýth an Impacted Permanent Central Incisor. J Tenn Dent Assoc 1997 Oct;77(4):46-8.

8. Hisatomi M, Asaumi J, Konouchi H, Honda Y, Wakasa T, Kishi K. A case of complex odontoma associated with an impacted lower deciduous second molar and analysis of the 107 odontomas. Oral Dis 2002 Mar;8(2):100-5.

9. Yildirim G, Tosun G, Kiziloglu D, Durmus E, Sener Y. An unusual association of odontomas with primary teeth. Eur J Dent 2007 Jan;1(1):45-9.

10. Yerli H, Konus O, Arac M, Cicek D. Case report: Complex composite odontoma: Plain radiography and computed tomography findings. Tani Girisim Radyol. 2003 Mar;9(1):97-9.

11. Sikes JW, Ghali GE, Troulis MJ. Expansile intraosseous lesion of the maxilla. J Oral Maxillofac Surg 2000;58:1395-400.
12. Hidalgo O, Leco M, Martínez J. Metaanalysis of the epidemiology and clinical manifestations of odontomas. Med Oral Patol Oral Cir Bucal 2008 Nov 1;13(11):730-4.

13. Amado Cuesta S, Gargallo Albiol J, Berini Aytés L, Gay Escoda C. Review of 61 cases of odontoma. Presentation of an erupted complex odontoma. Med Oral 2003 Nov;8(5):366-73.

14. Dinatale E. Neuralgia sintomática de la tercera rama del trigémino asociada a un odontoma compuesto. Presentación de un caso. Acta Odontol Venez 2003;41(3):50-3.

15. Junquera L, Vicente J, Roig P, Olay S, Rodríguez O. Intraosseous odontoma erupted into the oral cavity: an unusual pathology. Med Oral Patol Oral Cir Bucal 2005 May-Jul;10(3):248-51.

16. Chindasombatjaroen J, Kakimoto N, Akiyama H, Kubo K, Murakami S, Furukawa S, et al. Computerized tomography observation of a calcifying cystic odontogenic tumor with an odontoma: case report. Oral Surg Oral Med Oral Pathol Oral Radiol Endod 2007 Dec; 104(6):e52-7. Epub 2007 Oct 17.

17. Serra-Serra G, Berini-Aytés L, Gay-Escoda C. Erupted odontomas: a report of three cases and review of the literature. Med Oral Patol Oral Cir Bucal 2009 Jun 1;14(6):E299-303.

18. Ilief-Ala MA, Eisenberg E, Mathieu G. Peripheral complex odontoma in a pediatric dental patient: a case report. J Mass Dent Soc 2008 Winter; 56(4):24-6.

19. Hirshberg A, Kaplan I, Buchner A. Calcifying odontogenic cyst associated with odontoma: a possible separate entity (odontocalcifying odontogenic cyst). J Oral Maxillofac Surg 1994 Jun;52(6):555-8.

20. Gallana-Álvarez S, Mayorga-Jimenez F, TorresGómez FJ, Avellá-Vecino FJ, Salazar-Fernández C. Calcifying odontogenic cyst associated with complex odontoma: case report and review of the literature. Med Oral Patol Oral Cir Bucal 2005 May-Jul;10(3):243-7. 
21. Delgado W, Arrascue M, Calderón V, Paniura D. Hybrid Oodontogenic Tumour: calcifying cystic odontogenic tumor with complex odontoma and foci of ameloblastoma. Rev Estomatol Herediana 2006 Apr; 16(2):120-125.

22. Nelson-Filho P, Silva RA, Faria G, Freitas AC. Odontoma-like malformation in a permanent maxillary central incisor subsequent to trauma to the incisor predecessor. Dent Traumatol 2005 Oct;21(5):309-12.

23. Widad FA, Putrus ST. Erupted odontoma. Oral Surg Oral Med Oral Pathol 1985;59:225-6.

24. Chang JY, Wang JT, Wang YP, Liu BY, Sun A, Chiang CP. Odontoma: a clinicopathologic study of 81 cases. J Formos Med Assoc 2003 Dec;102 (12):876-82

25. Amailuk P, Grubor D. Erupted compound odontoma: case report of a 15-year-old Sudanese boy with a history of traditional dental mutilation. $\mathrm{Br}$ Dent J 2008 Jan 12;204(1):11-4.

26. Ide F, Shimoyama T, Horie N. Gingival peripheral odontoma in an adult: case report. J Periodontol 2000;71:830-2.

27. Kaplan I, Nicolaou Z, Hatuel D, Calderon S. Solitary central osteoma of the jaws: a diagnostic dilemma. Oral Surg Oral Med Oral Pathol Oral Radiol Endod 2008 Sep;106(3):e22-9. Epub 2008 Jul 7.

28. Singer SR, Mupparapu M, Milles M, Rinaggio J, Pisano D, Quaranta P. Unusually large complex odontoma in maxillary sinus associated with unerupted tooth. Report of case and review of literature. N Y State Dent J 2007 Jun-Jul;73(4): 51-3.

29. da Silva LF, David L, Ribeiro D, Felino A. Odontomas: a clinicopathologic study in a Portuguese population. Quintessence Int 2009 Jan;40(1): 61-72.

30. Delbem A, Cunha R, Bianco K, Afonso R, Gonçalves T. Odontomas in pediatric dentistry: report of two cases. J Clin Pediatr Dent 2005 winter;30(2):157-60.

31. Kaneko M, Fukuda M, Sano T, Ohnishi T, Hosokawa Y. Microradiographic and microscopic investigation of a case of complex odontoma. Oral Surg Oral Med Oral Pathol Oral Radiol Endod 1998;85:131-4.

32. García-Consuegra L, Junquera LM, Albertos JM, Rodríguez 0. Odontomas. A clinical-histological and retrospective epidemiological study of 46 cases. Med Oral 2000 Nov;5(5):367-372.

33. Ochsenius G, Ortega A, Godoy L, Peñafiel C, Escobar E. Odontogenic tumors in Chile: a study of 362 cases. J Oral Pathol Med 2002 Aug;31(7): 415-20.

34. Lukes SM, Wachter KM. Compound odontoma: a case study. J Dent Hyg 2003 Winter;77(1): 47-9.

35. Buchner A, Merrell PW, Carpenter WM. Relative frequency of central odontogenic tumors: a study of 1,088 cases from Northern California and comparison to studies from other parts of the world. J Oral Maxillofac Surg 2006 Sep;64(9): 1343-52.

36. K. Yeung, R. Cheung, M. Tsang. Compound odontoma associated with an unerupted and dilacerated maxillary primary central incisor in a young patient. Int J Paediatr Dent 2003; 13: 208212.

37. Adebayo ET, Ajike SO, Adekeye EO. A review of 318 odontogenic tumors in Kaduna, Nigeria. $\mathrm{J}$ Oral Maxillofac Surg 2005 Jun;63(6):811-9.

38. Owens BM, Schuman NJ, Mincer HH, Turner JE, Oliver FM. Dental odontomas: a retrospective study of 104 cases. J Clin Pediatr Dent 1997 Spring; 21(3):261-4.

39. Miki Y, Oda Y, Iwaya N, Hirota M, Yamada N, Aisaki K, et al. Clinicopathological studies of odontoma in 47 patients. J Oral Sci 1999. Dec; 41(4):173-6. 
40. Vázquez Diego J, Gandini Pablo C, Carbajal Eduardo E. Compound odontoma: Radiographic diagnosis and surgical management of a clinical case. Av. Odontoestomatol 2008; 24 (5):307-312.

41. Tomizawa M, Otsuka Y, Noda T. Clinical observations of odontomas in japanese children: 39 cases including one recurrent case. Int $\mathrm{J}$ Paediatr Dent 2005 Jan; 15(1):37-43.

42. Litonjua LA, Suresh L, Valderrama LS, Neiders ME. Erupted complex odontoma: a case report and literature review. Gen Dent 2004 May-Jun; 52(3):248-51.
43. Faus Llacer VJ, Camps Alemany I, Pascual Moscardo A, Paricio Martin J. Diagnosis of compound odontoma. Apropos of 2 cases. Rev Eur Odontoestomatol 1990 Sep-Oct;2(5):325-8.

\section{CORRESPONDENCIA}

Jonathan Harris Ricardo.

Alpes transversal 74 \# $31 \mathrm{~g} 53$

Cartagena. D.T y C.

Colombia

Correo electrónico: j.harris.r@hotmail.com. 